\title{
Chantal Mouffe im Gespråch mit Michael Walzer
}

*Man muß nicht nur tolerant sein, sondern auch demütig*

Chantal Mouffe - Ihr Werk beginnt, in Frankreich bekannt zu werden. Es erregt lebhaftes Interesse, da es einen wenig verbreiteten Typus politischer Philosophie verkörpert. Sie sind sowohl Historiker als auch Theoretiker, Sie nehmen die Literatur und die Religion, Philosophie und Geschichte gleichermaßen zum Ausgangspunkt Ihrer Reflektionen. Außerdem verfolgen Sie die aktuellen politischen Ereignisse und scheuen sich nicht vor politischem Engagement. Daher die Vielfalt und der Reichtum Ihrer Eingriffe. In die angloamerikanische Debatte über Gerechtigkeit haben Sie auf vielbeachtete Weise eingegriffen Spheres of Justice, (deutsche Übersetzung Sphären der Gerechtigkeit, Campus-Verlag Frankfurt/Main 1992). Im Rahmen dieser Diskussion werden Sie generell in die Gruppe derjenigen Autoren eingeordnet, die die 'Communitarians' genannt werden, weil sie die individualistische Konzeption des Subjekts, wie man sie bei den liberalen Kantianern findet, im Namen einer visionåren 'konstitutiven' Gemeinschaft kritisieren. Doch haben Sie sich zunehmend von dieser Position entfernt. Ich ko̊nnte mir vorstellen, daß Sie sich im Grunde jenen Denkern nicht mehr verwandt fühlen, die wie Richard Rorty oder John Gray den politischen Liberalismus verteidigen wollen, indem sie Universalismus- und Rationalismuskritiken liefern und eine 'kontextualistische' Perspektive anbieten. Genau wie Sie betrachten jene die moderne Dernokratie als Tradition und lehnen jeden Versuch $\mathbf{a b}$, die demokratischen Werte metaphysisch zu begründen.

Michael Walzer - Wie Sie wissen, handelt es sich nicht um organisierte Gruppen oder gar um kohärente politische Tendenzen. Wenn man sie auf einer Karte situieren wollte, würde man eine Karte der akademischen Welt benötigen, nicht der politischen oder der 'realen' Welt. Deshalb habe ich es nicht gern, wenn man in meinen Paß 'Communitarian', 'Universalist' oder 'Postmoderner' schreibt. Das entspricht nicht meinen Einstellungen. Andererseits rede ich gern von dem, was ich bin, vielleicht manchmal zuviel: Amerikaner, Jude, Intellektueller, demokratischer Sozialist, etc. Da fühle ich mich engagiert, und da wurde mein 'Ich' konstituiert, und zwar mehrmals. Ich vermute, daß man mich nicht zufallig als Communitarian klassifiziert. Aber ich fühle mich unwohl mit dem kommunitären Eifer und der etwas erstickenden Idee, eine einzige Gemeinschaft könne unseren Bedürfnissen genügen. Ich kenne meine inneren Widersprüche und erkenne die gleichen Zeichen von Gespaltenheit an 
meinen Freunden wieder. Was wir brauchen, ist eine weniger beengende Politik, etwas wie eine liberale und pluralistische Sozialdemokratie. Ich will allerdings herausstreichen, daß wir für eine solche Politik kåmpfen müssen. Denn wenn sie ihren Ursprung in bestimmten Charakteristika unserer politischen Tradition hat, ist es notwendig, daß wir andere Charakteristika der gleichen Tradition bekämpfen. Mir liegt es fern, mich gemütlich in der Tradition und in den überlieferten Kontexten installieren zul wollen wie ein Greis im Lehnstuhl. Ich kann mir sehr wohl theoretische Grundlagen ausmalen, aber das hålt mich nicht davon $a b$, die Einheit zwischen Theoriekritik und politischer Praxis zu suchen.

Chantal Mouffe - Obwohl man Sie of John Rawls entgegensetzt, habe ich den Eindruck, daß Sie ihm in Wirklichkeit auf politischer Ebene näher sind als seinen Kritikern aus den Reihen der communitarians, da auch Rawls eine bestimmte Form der Sozialdemokratie verteidigt. Seine theoretische Position hat sich andererseits seit Eine Theorie der Gerechtigkeit (Suhrkamp-Verlag Frankfurt 1979) weiterentwickelt. In seinen neueren Artikeln versichert er, daß es nicht sein Ehrgeiz sei, eine Theorie der Gerechtigkeit zu formulieren, die für alle Gesellschaften gültig wäre. Gerechtigkeit im Sinne von Ausgleich (Équité), so Rawls, stützt sich auf intuitive Vorstellungen in der politischen Kultur der liberal-demokratischen Gesellschaften; sie sind daher nur für diese Gesellschaften gültig. Ist das nicht eine Perspektive der 'kontextualistischen' Art, die der Ihren ganz ähnlich ist, auch wenn die Resultate, die Sie beide erzielen, verschieden sind?

Michael Walzer - Es ist für mich keine Überraschung festzustellen, daß Rawls einige Schritte in meine Richtung gemacht hat. Doch habe ich den Eindruck, daß es auch auf Seiten der 'Kontextualisten' Ånderungen gegeben hat: gewisse Versuche, die Gemeinsamkeiten, die es zwischen allen oder fast allen - menschlichen Zusammenhängen gibt, hervorzuheben. Ich möchte beispielsweise meine Solidarität mit den tschechischen oder chinesischen Demokraten ausdrücken können, auch wenn diese die ins Augen springenden Affinitäten zwischen ihren Argumenten und der amerikanischen Erfahrung nicht akzeptieren. So nähem wir uns beide - an was an? An einen immer noch ungenügend definierten Punkt. Nichtsdestotrotz bleiben noch genug wichtige und augenfallige Unterschiede zwischen uns bestehen, vielleicht wegen der akademischen Disziplinen, die unsere jeweiligen Konzeptionen der distributiven Gerechtigkeit geprägt haben. Rawls läßt sich von theoretischer Ökonomie und Entwicklungspsychologie inspirieren, ich mich von Geschichte und Anthropologie. Daraus folgt, daß sich in seiner 'Gerechtigkeit als Verteilungsgerechtigkeit' nur ein Komplex kohårenter Prinzipien findet, während die distributiven Prin- 
zipien meiner 'komplexen Gerechtigkeit' radikal anders sind, eher in Harmonie mit unseren Vorstellungen von sozialen Güter als mit den Resultaten eines rationalen Wahlverfahrens.

Chantal Mouffe - Kommen wir kurz auf Thre Vision einer pluralistischen und liberalen Sozialdemokratie zurück. Mit diesem Projekt identifiziere auch ich mich und habe versucht, es auszuarbeiten, ausgehend von der Idee einer 'Mehrheitsdemokratie'. Diese beruht darauf, 'linke' Politik in Richtung einer 'Radikalisierung der Demokratie' zu überdenken, etwa mit dem Ziel der Erweiterung des Egalitätsprinzips und der Freiheit in einer zunehmenden Anzahl sozialer Beziehungen. In der Tat handelt es sich um verschiedene Modalitäten dessen, was Norberto Bobbio als 'liberalen Sozialismus' bezeichnet. Heute sind wir schon sehr viele, die finden, daß dies die einzig plausible und legitime Form darstellt, die Forderungen nach sozialer Gerechtigkeit mit der Achtung der politischen Institutionen einer liberal-demokratischen Ordnung zu verbinden. Angesichts der neoliberalen Welle in allen Ländern ist es dringlicher denn je, einem derartigen Projekt Form zu geben. Aus dieser Perspektive heraus haben mich Ihre Vorschläge eines 'Projekts der kritischen Assoziierung' besonders interessiert. Sie explizieren die oft von Ihnen verteidigte Idee eines Liberalismus, dessen innere Dynamik zum demokratischen Sozialismus führt. Michael Walzer - Ich habe immer geglaubt, daß ein demokratischer Sozialismus die Entwicklung eines reichen und von vielfältigen Beziehungen gestalteten Lebens gestatten müßte. Zweifellos sind freiwillige Verbindungen charakteristisch für die liberale Gesellschaft; ihre Verbreitung und Stärke stellen, zusammen mit der Idee von der eingeschränkten Regierung, das große Verdienst des Liberalismus dar. Doch waren diese immer auf bestimmte Klassen beschränkt; die große Mehrheit der Freiwilligen, die Aktiven an der Basis genauso wie die Führer, stammen aus der Mittel- und Oberschicht. Sie wiederholen oft im gesellschaftlichen Raum Formen der Herrschaft, die dem Raum von Markt und Staat entstammen. Die Volksmassen sind passiv und ängstlich, es sei denn, sie sind in der einen oder anderen linken Bewegung organisiert - in der Arbeiterbewegung, zur Verteidigung der bürgerlichen Rechte, als Feministen, etc.. Diese Mobilisierung quer durch die verschiedenen Bewegungen ist sehr wichtig und hat immer etwas Erregendes, aber man muß sich trotzdem fragen, was auf der Ebene der Institutionen davon übrigbleibt. Ich begreife die zivile Gesellschaft als eine Domäne, wo Engagement und Aktivismus infolge der alltåglichen Ůbernahme von Verantwortung, die nötig ist, damit es ein echtes öffentliches Leben geben kann, institutionalisiert und transformiert werden könnten. Erst im öffentlichen Leben können Kooperation, Beistand und Solidarität real und konkret werden. Wohlge- 
merkt, die zivile Gesellschaft braucht einen politischen Rahmen, und er muß vom Staat unterstützt werden; deshalb müssen ihre Mitglieder auch Staatsbürger sein. Aber es kommt mir so vor, als wäre die entscheidende Probe auf den Sozialismus seine Fähigkeit, die Gesellschaft selbst als eine kontinuierliche Schöpfung gewöhnlicher Männer und Frauen zu gestalten. Chantal Mouffe - Man entdeckt in Frankreich gerade die Rolle des Staates wieder. Nachdem man die zivile Gesellschaft vielleicht zu sehr privilegiert hatte, wird man sich nun bewußt, wie weit sie auf den Staat gründet. Daher eine Rückkehr des Politischen, das man tendenziell in seiner Entscheidungsdimension vernachlåssigt hat, wie auch das Thema Staatsbürgerschaft, dem wieder Ehre erwiesen wird und das, wie Sie gerade angedeutet haben, auf einer anderen Reflexionsebene angesiedelt werden muß. Denn in unserer Eigenschaft als Mitglieder einer politischen Gemeinschaft sind wir Staatsbürger. Dabei handelt es sich um einen allgemeineren Typus der Assoziierung, der vor anderen einen gewissen Vorrang haben sollte. Ich schätze, daß die Verständigung zwischen diesen unterschiedlichen Arten von Assoziierungen heute die strategische Frage bestimmt.

Michael Walzer - Ich bin einverstanden, was die Bedeutung des Staats betrifft, weil er Allgemeinheit auf einer Ebene darstellt, auf die keine der Gruppen der zivilen Gesellschaft hoffen kann. Wir können seine Rolle auf zwei Arten begreifen. Erstens, wenn Massenbewegungen die traditionellen Herrschaftsformen aufbrechen, braucht man den Staat, um die Veränderungen zu ratifizieren und ihre Wirksamkeit sicherzustellen - wie es während der US-amerikanischen Revolution der bürgerlichen Rechte der Fall war. Zweitens braucht man den Staat, um die neuen Formen der Freiheit und des Pluralismus (rechtlich und materiell) zu garantieren. Weil jene immer ein gewisses $\mathrm{Ma} \beta$ an Unbestimmtheit haben (das ist in der Tat sowohl für die Freiheit wie für den Pluralismus charakteristisch), wird die Rolle des Staats immer umstritten sein. Also muß man diese Fragen auf politische Art entscheiden, das heißt etwa, durch Vermittlung der engagierten und aufmerksamen Staatsbürger. Die Gesamtheit der Staatsbürger, die in allen staatlichen Institutionen agierende politische Gemeinschaft, muß über die Lősung dieser Konflikte entscheiden. Folglich sind diese aktiven Staatsbürger zur gleichen Zeit in den unterschiedlichen Gruppen der zivilen Gesellschaft engagiert. In welchem Maß sie sich jeweils engagieren sollten, ist eine Frage, die nicht theoretisch, sondern ausschließlich praktisch entschieden werden kann.

Chantal Mouffe - In der Anerkennung des Pluralismus liegt die Besonderheit der modernen Demokratie, und es ist nicht verwunderlich, daß das einen zenralen Raum in Ihrer Forschung einnimmt. Jedoch hat dieser 
Terminus derart verschiedene Bedeutungen, daß es nicht immer leicht zu verstehen ist, was Sie genau unter 'Pluralismus' verstehen und welche Rolle Sie ihm zuschreiben. Zahlreiche Verwirrungen sind der Tatsache geschuldet,daß dieser Begriff in der amerikanischen Politikwissenschaft sehr spezifisch gebraucht wird. Wie ordnen Sie sich den verschiedenen Typen von Pluralismus zu?

Michael Walzer - In meiner Arbeit findet man den Pluralismusbegriff mit zwei Hauptbedeutungen, die nicht immer ausreichend unterschieden werden. Da ist zunächst ein Pluralismus, der sich auf soziale Güter und auf die 'Sphären der Gerechtigkeit' bezieht, die diese konstituieren, mit ihren verschiedenen Verteilungsprinzipien und den ihnen korrespondierenden Prozeduren. Dann ist da schließlich der Pluralismus der sozialen Identitäten und ethnischen und religiösen Kulturen, die ihn erzeugen und begründen. Diese Form des Pluralismus muß man akzeptieren und sich auf sie einstellen, weil sie jeder modernen und komplexen Gesellschaft inhärent ist. Man kann sie unterdrücken - zum Preis der Tyrannei und Brutalität - aber man kann sie niemals eliminieren. Der Pluralismus, den Sie mit der amerikanischen politischen Wissenschaft identifizieren und der noch aus den sechziger Jahre stammt, ist einseitiger und apologetischer. Er suggeriert, daß die Macht in der amerikanischen Gesellschaft radikal zersplittert und verstreut sei: es gibt keinen Souverån, kein Zentrum, keine dominante Klasse, keine Elite an der Macht; nichts als liberale Staatsbuirger in einer Reihe von Gruppen organisiert, die sich ausgleichen und ihre demokratischen Rechte ausüben. Das ist eine These, die mit gewisser Vorsicht untersucht werden muß, weil sie teilweise wahr, aber insgesamt falsch ist. Außerdem ist es nicht evident, daß Zersplitterung ein demokratischer Wert sei. Natürlich wünschen wir uns manchmal, daß die Macht geteilt sei; aber das Ziel aller emstzunehmenden politischen Bewegungen ist, Macht zu erringen und zu sichern, um sie zu gebrauchen (auch wenn das nicht als 'Machtergreifung' verstanden werden muß).

Chantal Mouffe - Ihr Insistieren auf der Notwendigkeit, dem Pluralismus der Kulturen und Identitäten Raum zu schaffen, hat Ihnen oft den Vorwurf des 'Relativismus' eingetragen, unter anderem von Ronald Dworkin in seiner Polemik anläßlich Thres Buchs Spheres of Justice. Nach Dworkin müßte der Zweck einer Theorie der Gerechtigkeit die Begründung von immer und überall gültigen Prinzipien sein. Sie hingegen versichern, die politische Philosophie müsse 'in der Höhle bleiben'1 und ihre Rolle sei es, den Mitbürgem die Welt der Bedeutungen, die ihnen gemein

1 Damir wird auf das Höhlengleichnis Platons angespielt: Von der Höhle aus, mit dem Rücken zum Licht betrachtet, erscheinen an der Rückwand der Höhle alle Menschen und Gegensiände ununterscheidbar als Schatten 
sind, zu interpretieren. Dworkin vermutet, daß Sie das auf den Partikularismus festlegt und Sie letztlich kritikunfähig macht. Dennoch scheint mir Ihre Konzeption, obwohl 'kontextualistisch', nicht inkompatibel mit der Billigung bestimmter universeller Werte zu sein.

Michael Walzer - Gewiß, ich favorisiere eine Konzeption, die die Relativität der distributiven Gerechtigkeit beståtigt. Das Argument der Spheres of Justice lautet knapp und präzis: die Verteilung der sozialen Güter ist an die Bedeutung geknüpft, die diese im Leben der Menschen haben, an die man sie verteilen wird. Wie können wir über die Zuteilungen des Gesundheitswesens entscheiden, ohne uns eingehend mit dem der Gesundheit und langem Leben in einer bestimmten Gruppe zugeschriebenen Wert zu beschäftigen? Oder wie können wir eine Bildungspolitik begründen, ohne dem Stellenwert der Bildung in einer bestimmten Gesellschaft Aufmerksamkeit zu schenken? Aber die distributive Gerechtigkeit ist nicht das Ganze der Moral, das deckt nicht einmal das Gesamte der Gerechtigkeit ab. Als ich über gerechte und ungerechte Kriege schrieb, habe ich mich höchst universalistisch auf allgemeine Prinzipien bezogen, weil die Kriege zwischen Gesellschaften stattfinden und Fragen ins Spiel bringen, die über die kulturellen Grenzen hinausgehen. Zum Beispiel muß der entscheidende Gedanke von der Immunität der Nichtkämpfenden in wechselseitiger Anerkennung einer gemeinsamen Menschlichkeit verwurzelt sein - auch wenn das dann in verschiedenen Sprachen ausgedrückt werden wird. In Europa und Amerika sprechen wir wahrscheinlich vom Recht auf Leben und Freiheit. Das Selbstbestimmungsrecht der Voolker stellt, so glaube ich, eine kollektive Version dieses gleichen Rechts dar. Wenn ich also den Gegenstand der distributiven Gerechtigkeit verhandle, appeliere ich an die Bedeutungen und an die Umgangsweisen damit in meiner eigenen Gesellschaft (die liberal und demokratisch ist). Aber ich werde zum Thema der vietnamesischen und irakischen Bürger ebenso wenig schweigen, wie zu den Kurden, Palästinensem und Tibetanem, die ich kaum kenne und deren Lebensweise anders als meine ist. Im Unterschied $\mathrm{za}$ Dworkin maße ich mir nicht an, ihnen zu sagen, wie sie die gute Gesellschaft organisieren müssen; ich möchte ihnen nur die Möglichkeit geben, es selbst zu machen. Wenn sie es wirklich sehr schlecht machen, dann werde ich mich Dworkin anschließen, um sie zu kritisieren.

Chantal Mouffe - Man muß in der Tat das unglückliche Dilemma zwischen Relativismus und Universalismus aufsprengen. Entgegen dem, was 'Universalisten' wie Habermas, Dworkin oder Ackerman behaupten, läßt sich eine Perspektive vermitteln, die einereits auf der Unmo̊glichkeit insistiert, vom fernen Stern Sirius aus die Welt zu betrachten, und andererseits auf der Notwendigkeit beharrt, im Denken immer von einer festge- 
legten Tradition auszugehen und universale Werte $\mathrm{zu}$ verteidigen - soweit sie universalisierbar sind. Anstatt sich in sterile Debatten verstricken zu lassen, muß man, glaube ich, ein neues Verhältnis zwischen dem Allgemeinen und dem Besonderen erstreben.

Michael Walzer - In der Tat. Übrigens glaube ich, daß das Festhalten am Pluralismus der sozialen Güter und der kulturellen Identitäte partikularistisch und universalistisch zugleich ist. Die Existenz des Unterschieds gilt es anzuerkennen - überall. Die Anerkennung ist universal, während das, was anerkannt wird, lokal und partikular ist. Man könnte das als einen 'reiterativen' Universalismus bezeichnen. Die Güter und Identitåten werden ständig, doch nie auf die gleiche Art und Weise erzeugt. Es kommt darauf an, den Wert der Kreativität zu schätzen und ihre Produkte zu respektieren. Aber es ist auch nötig, diesen sozialen Schöpfungsprozessen gewisse Grenzen zu setzen; ihre Protagonisten müssen mit denen der anderen Prozesse in Frieden leben, und sie dürfen nicht versuchen, ihnen ihre eigenen Konzeptionen von Politik oder Kultur aufzuzwingen. Autoren wie Habermas oder Ackerman meinen von diesen Einschränkungen ausgehend, daß es möglich sein müßte, die verschiedenen Schöpfungsprozesse alle zu einem einzigen und gleichen Resultat auf der Grundlage einer universellen Moral zu leiten. Um das zu erreichen, braucht man radikale Beschränkungen, die wir nicht etablieren können, ohne vorher zu wissen, was das Resultat sein sollte - und immer sein sollte. Nun ist das ein Wissen, das uns nicht zugänglich ist. Ich verteidige hier nicht nur die Toleranz, sondern den Gedanken, daß alle menschlichen Dinge notwendig partial und unvollständig sind. Es gibt ein altes jüdisches Sprichwort über Gottes Gesetz: *Wende es und wende es noch einmal, alles ist in ihm*. Vielleicht. Aber wir werden nie fähig sein, 'alles' daraus zu abzuleiten. Wir leben immer mit etwas weniger, und für alle anderen ist es ebenso das etwa wird die Art sein, wie sie es wenden.

Chantal Mouffe - Ein häufig gegen eine Perspektive, die den Akzent auf Pluralismus der Traditionen setzt, vorgebrachtes Argument lautet, daß sie es nicht erlaube, die allgemeinen Menschenrechte zu begründen. Diese Perspektive präsentiert die Menschenrechte als Produkt einer bestimmten Tradition, als eine spezifisch okzidentale Angelegenheit, während man sie als Ausdruck eines 'moralischen Fortschritts' betrachten müßte, dessen Evidenz von jedem vernunftbegabten Wesen hätte akzeptiert werden können. Eine solche allgemeine Begründung verleugnen zu wollen, könnte heißen, die Barbarei zu rechtfertigen.

Michael Walzer - Die Sprache der Menschenrechte ist nichts anderes als unsere besondere Weise, von bestimmten menschlichen Werten zu sprechen, die zentral und allgemein anerkannt sind. Wenn wir beispielsweise 
sagen, daß Idi Amin, Pol Pot oder Saddam Hussein (ich nehme hier evidente Fälle) die Menschenrechte vergewaltigt haben, beschuldigen wir sie für etwas, das auch als Brutalität oder Barbarei, inhumane Akte oder Verbrechen wider Gott hätte bezeichnet werden können. Ich gehe von dem Prinzip aus, daß unsere Anschuldigung in diese und andere Sprachen übersetzt werden kann. Weil sie übersetzt werden kann, kann sie jenseits unserer politischen und kulturellen Grenzen verstanden und angewandt werden. Doch wir hätten Unrecht, wenn wir meinen würden, daß die Sprache der Menschenrechte dazu dienen könne, den besonderen Charakteren unserer eigenen politischen Kultur Rechtsgültigkeit zu verleihen, so als ob alle menschlichen Wesen moralisch dazu bestimmt seien, auf die gleiche Weise wie wir zu leben, unter Ausschließung jeder anderen. Ich glaube nicht, daß man das Argument zugunsten des radikalen Individualismus durch eine Liste der individuellen Rechte stärkt. Je länger die Liste, desto weniger plausibel wird sie sein, desto eher wird sie der nur lokalen Bedeutung und dem lokalen Echo geweiht sein (unter den Individualisten, ihren Freunden und Familien).

Chantal Mouffe - Auch ich schätze, der Menschenrechtsdiskurs ist nicht unmittelbar in seiner okzidentalen Form universalisierbar, sondern muß in das Vokabular anderer Kulturen übersetzt werden, um akzeptiert und zugkräftig zu werden. Andererseits glaube ich, daß wir schnellstens den zufälligen und fragilen Charakter der Institutionen begreifen můssen, aus denen sich die moderne Demokratie herauskristallisiert hat. Diese Siege, weit davon entfernt, eine notwendige Etappe der Menschheitsgeschichte zu repräsentieren, einen Punkt ohne Wiederkehr, müssen von uns verteidigt und geschützt werden, da es gefährlich wäre, sie als von selbst entstandene Rechte anzusehen. Doch konsolidieren wir sie nicht, indem wir ihnen rationale Grundlagen verschaffen, sondern durch Vervielfachen der Praktiken, in die sie sich einschreiben können, sowie durch Absichern der verschiedenen Identitäten, die sie hatten. Tatsächlich kann eine starke Identifikation der Staatsbürger mit der demokratischen Tradition die beste Garantie dafür abgeben, daß diese überleben und sich vertiefen kann. Das ist eine Sache, die mir besonders am Herzen liegt, da ich befurchte, daß wir gerade eine Zeit erleben, in der diese Werte durch das Wiederaufleben alter ethnischer und religiöser Antagonismen einer harten Probe unterzogen werden. Hat nicht das Explodieren von Antisemitismus, Rassismus und Nationalismus, das wir überall wahrnehmen, gewisse etwas naive okzidentale Konventionen über Politik und den 'natürlichen' Charakter der Demokratie erschüttert?

Michael Walzer - Untersuchen wir diese Wiederkehr der alten Antagonismen, diesen zugleich alten und neuen Stammeskult. Die Linke ist aus 
den Stämmen nie schlau geworden. Es ist inzwischen klar, daß ein Großteil der Halsstarrigkeit, des passiven aber wirkungsvollen Widerstands, die diese totalitären Régimes des Ostens zerfressen haben, aus Leidenschaften und Loyalitäten hochgradig partikularistischer Natur kommen. Wir müssen uns über die Macht dieses Partikularismuses wundern. Er wurde in vielen Jahrzehnten, in vielen Generationen ohne irgendeine Unterstützung von offiziellen Institutionen wie Schulen oder Medien erzeugt. Ich stelle mir vor, daß allüberall Großeltern ihren Enkeln Dinge zuflüstern, ihnen Geschichten erzählen. Ich bin nicht dafür, einen politischen Kampf gegen diese Leute zu führen. Lassen wir sie ihre Geschichten öffentlich erzählen. Das, was an ihnen positiv ist, würde dadurch verstärkt, das Negative, das Fanatische und das, was nur Ressentiment ist, wäre der Kritik ausgesetzt. Diese Geschichten müssen sozusagen in Szene gesetzt werden, freilich nur insoweit als sie keinerlei Ungerechtigkeit gegen diejenigen, die andere Geschichten erzählen, provozieren. Dafür wird man viel Kreativität und politische Kunst brauchen, wie auch eine große Bandbreite institutioneller Voraussetzungen - Dezentralisierung, lokale Autonomie, Föderalismus, etc. Vielleicht wird das, was im Westen mit der Religion passiert ist, schließlich mit dem Nationalismus im Osten passieren: die Tatsache, daß Unterschiede sich Platz schaffen, wird nach und nach $\mathrm{Haß}$ und Fanatismus unterhöhlen. Auf alle Fälle scheint mir eines sicher: der Versuch, einen liberalen Sozialismus zu schaffen, muß an eine 'liberale' Lo̊sung der nationalen Frage geknüpft sein. Ich ahne, daß das auch von einem langen Prozess der Entzauberung der Ökonomie des freien Markts abhängt, der kaum angefangen hat. Aber das ist eine andere Geschichte.

Chantal Mouffe - Die Herausforderung, der wir uns im Osten genauso wie in Europa und in den USA konfrontiert sehen, ist die einer neuen Konzeption der Staatsbürgerschaft. Hier und dort stellt sich die Frage auf verschiedene Weise, aber letztlich geht es um das gleiche Problem: wie ist die Anerkennung des ethnischen, religiösen und kulturellen Pluralismus mit der Zugehörigkeit zu einer demokratischen politischen Gemeinschaft, deren politische Prinzipien die Bestätigung der Freiheit und der Gleichheit fuir alle sind, in Einklang zu bringen. Ich glaube, daß man die Staatsbürgerschaft als Identifikation mit diesen Prinzipien begreifen muß und nicht bloß als einen rechtlichen Status, als einen Komplex von Rechten. Der Versuch der 'Communitarians', den aktiven Aspekt der Staatsbürgerschaft wie man ihn im bürgerlichen Republikanismus findet, neu zu bestimmen, antwortet so betrachtet einem echten Bedürfnis. Das Problem ist, daß ihre Konzeption vom Gemeinschaft keinen Raum für Pluralismus läßt. Aber die liberale Vision, die die Demokratie ausschließlich als 
Komplex von Rechtsverfahren präsentiert, ist gänzlich unzureichend, weil die moderne Demokratie Werten nicht neutral gegenübersteht. Wenn wir nicht erreichen, daf ihre normative, ethisch-politische Dimension akzeptiert wird, bezweifle ich, daß wir fähig sein werden, den zentrifugalen Kräften des Partikularismus standzuhalten.

Michael Walzer - Im linken Denken findet sich oft die Idee, die in Wirklichkeit von Rousseau stammt, daß die Staatsbürgerschaft mit einem totalen und exklusiven Engagement verbunden sein muß; der Staatsbürger wird als ungeteilter Bürger, dessen Begeisterung für das Gemeinwesen durch eine homogene Kultur und eine zivile Religion garantiert wäre, gedacht. Aber eine solche Sicht der Dinge macht aus der Politik etwas zu Einfaches (in Wirklichkeit war Rousseau ein Feind des politischen Lebens: er war gegen dessen Aufspaltungen und Agitationen). Gefährlich daran ist vor allem, daß die politischen Führer die Vielfalt der Probleme, die sie in den zeitgenössischen Gesellschaften antreffen, unterdrücken und transformieren wollen. Mir scheint eher, daß wir die Staatsbürgerschaft als eine unserer Verpflichtungen unter anderen ansehen und ihr zugleich einen entscheidenden Stellenwert zuerkennen sollten, weil sie uns als Vermittlungsinstanz zwischen unseren anderen Verpflichtungen dient und es uns ermöglicht, über die verschiedenen Bereiche hinweg zu agieren. Die politische Gemeinschaft ist eine Domäne gemeinsamen Handelns hinsichtlich gemeinsamer Ziele. Diese Ziele decken nicht die Gesamtheit des guten Lebens ab; man findet in der Politik weder die Seligkeit, noch die Selbstverwirklichung, noch die Liebe. Nichtsdestotrotz ist es gut für Männer und Frauen, gemeinsam daran zu arbeiten, den verschiedenen Modalitäten ihrer Koexistenz Form zu geben; dem Sich-Treffen, dem Diskutieren, dem Überlegen und dem Entscheiden. Bei einer solchen Übung sind wichtige menschliche Fähigkeiten am Werk, und gerade die Schwierigkeiten, die man dabei erlebt und manchmal überwinden kann, sind es, die uns von ihrem Wert überzeugen müßten.

Chantal Mouffe - Folglich muf man wohl akzeptieren, daß eine demokratische, wirklich pluralistische Politik den relativen, prekären und unfertigen Charakter der Lo̊sungen für die Probleme unserer Koexistenz erdulden muß. Deshalb kann es in einer demokratischen Gesellschaft keine alleinige, definitive Antwort auf die Gerechtigkeitsfrage geben. Es wird immer verschiedene Auslegungen der Art, wie die Prinzipien der Freiheit und Gleichheit institutionalisiert werden müssen, geben, und soziale Beziehungen, auf die man sie bequem anwenden kann. Daher die Sinnlosigkeit der Behauptung, eines 'rationale' Lösung dieses Problems za haben. Michael Walzer - Das ist genau, was ich meine. Aus diesem Grund hat alles, was ich je über Gerechtigkeit und Sozialkritik geschrieben habe, 
zum Ziel, einen Rahmen für den Interpretationsprozeß und für das, was wir $\mathrm{zu}$ interpretieren haben, anzugeben. Meine Kritiker beklagen sich, daß ich keine endgültige Methode anbiete, um sich zwischen widerstreitenden Interpretationen zu entscheiden. Aber es gibt keine derartige Methode. Der Entscheidungsakt ist ein sozialer Prozeß - eine Mischung aus Argument, Rhetorik, Manipulation, Druck und Einschränkung. Die Aufgabe der Intellektuellen deckt nur einen Teil dieses Vorgangs ab; wir haben die besten Argumente, deren wir fähig sind, zu präsentieren, ohne zu behaupten, sie seien mehr als partial und unvollständig. Die Überzeugung, eine endgültige Lösung gefunden zu haben oder eine einzigartige, rationale Interpretation anbieten zu können, führt in der Tat zur Rechtfertigung des Zwangs. Man muß nicht nur tolerant sein, sondern auch demütig. Die Verbrechen der Linken im Lauf dieses Jahrhunderts haben viel mit intellektueller Arroganz zu tun. (Die Verbrechen der Rechten hingegen haben einen materialistischeren Ursprung: individuelle Habsucht und kollektiven Egoismus.)

Chantal Mouffe - Das ist genau der Grund, weshalb ich einem gewissen Typus politischer Philosophie mißtraue; jenem nämlich, der sich befleiBigt, unwiderlegbare Argumente für die Demokratie zu liefern, der sie mit der menschlichen Natur begründet oder mit universaler Vernunft. Ein derartiges Verlangen nach Zugang zu der rationalen Erklärung menschlicher Koexistenz ist Ausdruck eines gefährlichen Willens zur Macht, der dem Pluralismus, der für die moderne Demokratie grundlegend ist, entgegengesetzt ist. Dieser ist notwendig konfliktgeladen und schließt die Möglichkeit, einen definitiven Konsens zu erreichen, aus. Wir können Spaltungen und Antagonismen nicht entkommen, und es ist schon wahr, daß die Politik unser Schicksal ist. Ist es nicht paradox, daß die pluralistische Demokratie nur wegen der Unmöglichkeit ihrer Vollendung existieren kann?

Michael Walzer - Ja, Politik ist langwierig. Die Zusammenstöße zwischen Interessen, Werten und Überzeugungen finden kein Ende. Freiheit und Pluralismus intensivieren sie, anstatt sie zu beenden. Denn mehr Personen betreten die Arena; sie legitimieren eine größere Vielfalt von Interessen, Werten und Überzeugungen und sie trennen Macht und Autorität. Es ist möglich, daß wir mehr Freude am Konflikt haben werden (für einige ist das schon der Fall), wenn wir nicht dahin kommen, die offenkundigsten Formen der Unterdrückung zu beseitigen; auf alle Fälle wäre es besser, wir würden lernen, uns in diverse Auseinandersetzungen einzumischen. Das bringt mit sich, daß das Verlieren oder Siegen nicht total erfahren wird, daß Arroganz auf der einen und Demütigung und Furcht auf der anderen Seite nicht produziert werden. Ich sehe da eine dreifache Rolle 
für die politischen Philosophen: Erstens kommt es darauf an, Pluralismus und Unterschiede zu verteidigen, die damit verbundenen Konflikte zu akzeptieren, und gleichzeitig darauf $\mathrm{zu}$ bestehen, daß diese Konflikte immer partial bleiben: das Subjekt in seiner Gesamtheit, das Ganze der Gesellschaft dürfen nie in Frage gestellt werden. Zweitens müßten minimale Standards von Verfahren der Gerechtigkeit in der Politik augearbeitet werden, um die Grenzen unserer Streitigkeiten abzustecken - und um ' $\mathrm{Zi}$ vilität' und Anteilnahme zu verteidigen. Drittens sollten wir offen für $\mathrm{Ge}-$ schichten und Visionen dessen bleiben, was eine substantiellere Gerechtigkeit sein könnte, freilich unter der Bedingung, daß sie nicht den Anspruch totaler Vollendung erheben und sich genauso oder sogar mehr an die Imagination als an den Willen richten. Fundament dafür ist die Konsequenz, die wir aus unserer Erfahrung sowie aus theoretischen Erwägungen, die Sie in den Vordergrund stellen, ziehen müssen: daß es keinen endgültigen Sieg gibt. Unser Willen wird immer mit dem anderer rivalisieren, mit denen wir aber ohne Unterlaß eine anständige Form der Koexistenz suchen müssen.

Dieses Gespräch erschien zuerst in Esprit, Juni 1991. Übersetzung: Eva Liebendörfer 